\title{
Link!: Potential Field Guidance Algorithm for In-Flight Linking of Multi-Rotor Aircraft
}

\author{
John R. Cooper* \\ National Institute of Aerospace, Hampton, VA, 23666, USA \\ Paul M. Rothhaar ${ }^{\dagger}$ \\ NASA Langley Research Center, Hampton, VA, 23681, USA
}

\begin{abstract}
Link! is a multi-center NASA effort to study the feasibility of multi-aircraft aerial docking systems. In these systems, a group of vehicles physically link to each other during flight to form a larger ensemble vehicle with increased aerodynamic performance and mission utility. This paper presents a potential field guidance algorithm for a group of multi-rotor vehicles to link to each other during flight. The linking is done in pairs. Each vehicle first selects a mate. Then the potential field is constructed with three rules: move towards the mate, avoid collisions with non-mates, and stay close to the rest of the group. Once a pair links, they are then considered to be a single vehicle. After each pair is linked, the process repeats until there is only one vehicle left. The paper contains simulation results for a system of 16 vehicles.
\end{abstract}

\section{Introduction}

INK! is a multi-center effort at NASA to study groups of aerial vehicles with the capability of physically Llinking to each other to form a larger structure with improved aerodynamic performance and mission utility compared to the individual constituents. This paper establishes a guidance algorithm for in-flight linking of multi-rotor vehicles. Linking a large group of vehicles together is done in a systematic way by selecting pairs of vehicles, driving the pairs towards each other until every pair is linked, and then repeating this process until all vehicles are joined together. The main contribution of this work is the application of potential field theory for guidance during the linking process.

Linked aircraft have the potential to drastically increase the mission capabilities of an aircraft system. Such coordination could enable high altitude, long endurance (HALE) missions with vertical takeoff and landing of component vehicles from unimproved sites. The same system could reconfigure to be capable of a heavy lift mission. Furthermore, in-flight linking allows for multi-leg missions that consist of efficient flight to a remote location followed by a distributed multi-agent leg.

Linked aircraft may find applications in missions such as search and rescue, cartography, reconnaissance, package delivery, and atmospheric satellites. For observational missions, the aircraft would fly to the desired location in an efficient, large fixed-wing mode. Then, the system would break apart into its individual units to cover ground in an optimal distributed way. In the case of the persistent atmospheric satellite mission, units would break off for refueling and immediately be replaced by a new aircraft. In this manner, the ensemble craft could stay airborne indefinitely. Additionally, HALE vehicles have high wing aspect ratios making them sensitive to atmospheric turbulence. This is what caused the crash of NASA's Helios prototype. ${ }^{1}$ A

\footnotetext{
* Research Engineer, Research Department, 100 Exploration Way, Hampton, VA 23666, AIAA Student Member.

${ }^{\dagger}$ Aerospace Technologist, Dynamic Systems and Control Branch, 11 Langley Blvd, Hampton, VA 23681, and AIAA Member.
} 
system of linked aircraft would be capable of breaking off into individual agents during turbulence and then re-linking once it is safe to do so.

Flight testing of linked aircraft was first performed in the 1950's by the US Air Force with projects TipTow $^{2}$ and Tom-Tom. ${ }^{3}$ These projects were carried out in parallel with the objective of increasing the range of fighter planes. Both involved linking the wingtips of the fighters to the wingtips of a larger bomber aircraft. Several successful flights were performed during both projects. However, introducing an autopilot to Project TipTow resulted in a failure which caused the loss of two planes and their crews. Project TomTom was canceled as a result of the link separating during flight due to wingtip vortices, although all crew members landed safely.

In the intervening years, research has been done on similar ideas such as the multibody transport concept by Moore and Maddalon which consists of two passenger jet fuselages joined together by a wing between them. ${ }^{4}$ This design allowed for a lower weight than two individual planes, due to reduced bending moment acting on the common wing and had a lift-to-drag ratio increase of $8-10 \%$. Magill et. al. later studied the effects of wingtip-docked flight. A method for stability analysis was given in ${ }^{5}$ and a $20-40 \%$ performance increase over individual flight was shown in. ${ }^{6}$

With modern day increased capability of unmanned systems and control hardware, there has been renewed interest in this problem. Several researchers have studied modular robotics over the last decade and a half. ${ }^{7-10}$ These systems consist of many small, often cube-shaped robots that are capable of arranging themselves in various configurations to complete different tasks. Oung et. al. extended this concept to aerial robots by developing an experimental platform known as the Distributed Flight Array. ${ }^{11}$ This array consists of many single rotor aircraft that dock with each other on the ground to form an arbitrarily-shaped multi-rotor vehicle. The individual craft are, however, unstable in solo flight.

More recently, Montalvo and Costello investigated flight dynamic modes for linked fixed-wing aircraft in wingtip-docked, nose-to-tail docked, and lattice configurations. ${ }^{12}$ Troub and Montalvo have further analyzed the controllability of these configurations in. ${ }^{13}$ Montalvo's dissertation addresses other aspects of the problem including linkage mechanism design and controller design. ${ }^{14}$

The linking algorithm presented in this paper results in $\log _{2}(n)$ linking phases, where $n$ is the number of vehicles in the group. Once the mating pairs are selected, a potential field is constructed which drives each vehicle to its mate while avoiding collisions with other agents and also keeping the total group of agents at a reasonable distance from each other. These goals are analogous to two of the three basic rules of flocking developed in ${ }^{15}$ and presented in: ${ }^{16}$

1. Flock centering - ensure that all agents remain close together

2. Obstacle avoidance - ensure that no agents collide with each other

3. Velocity matching - causes agents to move in the same direction and at the same speed

In the potential field guidance algorithm for in-flight linking, we remove the velocity matching rule, as this is not a requirement for linking. The obstacle avoidance rule is present for all agents except the mate agent, and the flock centering rule exists in its traditional form. In addition to these rules, we have added a rule for mate-linking.

The control input is computed by finding the steepest descent of the potential field. Potential field gradient algorithms were developed for flocking in body of work comprised of ${ }^{16-2019}$ introduced a differentiable, nonnegative, and radially unbounded potential field to represent the distance between two agents. The potential field developed for in-flight linking also has all of these properties to meet the prescribed goals.

The paper is organized as follows: Section II explains the multi-rotor vehicle dynamics. Section III presents the algorithm for mate selection, defines the potential field using the desired rules, and gives the control law to govern each agent. Section IV provides simulation results for a system of 16 vehicles. Finally, conclusions and future work are discussed in Section V 


\section{Multi-rotor Aerial Vehicle Dynamics}

Consider a multi-agent system composed of $n$ multi-rotor vehicles. The goal is for each vehicle to find a mate in the group, and physically link to this mate without colliding with other agents. The linked vehicles are then considered a single agent.

For the purposes of this paper, we will consider that each vehicle has an altitude-hold controller which holds all vehicles to the same altitude. Therefore, we will model the vehicle dynamics in $2 \mathrm{D}$. We will represent the dynamics of each vehicle in the plane of flight as

$$
\ddot{r}_{i}(t)=u_{i}(t) \quad i=1,2, \ldots, n
$$

where $r_{i} \in \mathbb{R}^{2}$ is the position of agent $i, u_{i} \in \mathbb{R}^{2}$ is the control input of agent $i$, and $t$ is time. Note that the control input is an acceleration, which can be controlled by tilting the vehicle such that the vertical component of the thrust vector is equal to the vehicle weight, and the horizontal components are equal to the desired accelerations times the vehicle mass. This can be expressed as

$$
\left[\begin{array}{c}
F_{x} \\
F_{y} \\
F_{z}
\end{array}\right]=R_{B}^{I}\left[\begin{array}{c}
0 \\
0 \\
T
\end{array}\right]
$$

where $F_{x}, F_{y}$, and $F_{z}$ are the $x, y$, and $z$-components of the vehicle thrust in the inertial frame, $R_{B}^{I}$ is the rotation matrix from the vehicle body frame to inertial frame, and $T$ is the vehicle thrust. $R_{B}^{I}$ is given in ${ }^{21}$ by

$$
R_{B}^{I}=\left[\begin{array}{ccc}
c_{\theta} c_{\psi} & s_{\phi} s_{\theta} c_{\psi}-c_{\phi} s_{\psi} & c_{\phi} s_{\theta} c_{\psi}+s_{\phi} s_{\psi} \\
c_{\theta} s_{\psi} & s_{\phi} s_{\theta} s_{\psi}+c_{\phi} c_{\psi} & c_{\phi} s_{\theta} s_{\psi}-s_{\phi} c_{\psi} \\
-s_{\theta} & s_{\phi} c_{\theta} & c_{\phi} c_{\theta}
\end{array}\right]
$$

where $s$ and $c$ denote sine and cosine of the subscripted variable respectively, $\phi$ is the vehicle's roll angle, $\theta$ is the pitch angle, and $\psi$ is the yaw angle. Note that the body $x$-axis points forward, the body $z$-axis points downward, and the body $y$ axis completes the triad.

To achieve a desired horizontal acceleration, $u_{i}=\left[u_{x} u_{y}\right]^{\top}$, we want

$$
\begin{aligned}
& F_{x}=m u_{x} \\
& F_{y}=m u_{y} \\
& F_{z}=-m g
\end{aligned}
$$

where $m$ is the vehicle mass and $g$ is the acceleration due to gravity. Substituting (4), (5), (6), and (3) into (2) allows one to solve for a roll angle, $\phi$, pitch angle, $\theta$, and a thrust, $T$ to provide the acceleration, $u_{i}$ for a given yaw angle, $\psi$.

We can obtain the required attitude command by solving the system of equations composed of (2) and (3). We immediately determine that $T=-\sqrt{F_{x}^{2}+F_{y}^{2}+F_{z}^{2}}$ because $R_{B}^{I}$ does not change the magnitude of a vector that it multiplies. The negative sign comes from the fact that the vehicle thrust vector always points in the negative body $z$-axis (upward). Let the solutions for $\phi$ and $\theta$ be $\phi_{c m d}$ and $\theta_{c m d}$ respectively. Solving (2) and (3) then yields

$$
\begin{aligned}
& \phi_{c m d}=\sin ^{-1}\left(\frac{F_{x}}{T} \sin (\psi)-\frac{F_{y}}{T} \cos (\psi)\right) \\
& \theta_{c m d}=\sin ^{-1}\left(\frac{\frac{F_{x}}{T}-\left(\frac{F_{x}}{T} \sin (\psi)-\frac{F_{y}}{T} \cos (\psi)\right) \sin (\psi)}{\cos \left(\sin ^{-1}\left(\frac{F_{x}}{T} \sin (\psi)-\frac{F_{y}}{T} \cos (\psi)\right)\right) \cos (\psi)}\right)
\end{aligned}
$$


The rotational equations of motion for a multi-rotor aircraft are given by the general rigid body dynamics,

$$
\begin{aligned}
& {\left[\begin{array}{c}
\dot{p} \\
\dot{q} \\
\dot{r}
\end{array}\right]=I^{-1}\left\{-\left[\begin{array}{l}
p \\
q \\
r
\end{array}\right] \times\left(I\left[\begin{array}{l}
p \\
q \\
r
\end{array}\right]\right)+\left[\begin{array}{l}
\tau_{1} \\
\tau_{2} \\
\tau_{3}
\end{array}\right]\right\}} \\
& {\left[\begin{array}{c}
\dot{\phi} \\
\dot{\theta} \\
\dot{\psi}
\end{array}\right]=\left[\begin{array}{ccc}
1 & s_{\phi} t_{\theta} & c_{\phi} t_{\theta} \\
0 & c_{\phi} & -s_{\phi} \\
0 & s_{\phi} / c_{\theta} & c_{\phi} / c_{\theta}
\end{array}\right]\left[\begin{array}{l}
p \\
q \\
r
\end{array}\right]}
\end{aligned}
$$

where $p, q$, and $r$ are the body-axis roll, pitch, and yaw rates respectively, $I$ is the vehicle's moment of inertia matrix, $\tau_{1}, \tau_{2}$, and $\tau_{3}$ are the body-axis torques about the $x, y$, and $z$ body axes respectively, and $t$ denotes the tangent of the subscripted angle. The body-axis torques and the thrust, $T$ comprise the control inputs for a multi-rotor aircraft.

\section{Potential Field Guidance Algorithm}

Let the mate for agent $i$ be agent $j$. The objectives of the guidance algorithm are to

1. Select $n / 2$ pairs, $(i, j)$, uniquely, i.e. agent $i$ pairs only to agent $j$, and agent $j$ pairs only to agent $i$.

2. Design $u_{i}$ such that agent $i$

(a) is driven towards agent $j$

(b) avoids all agents other than $j$

(c) is driven towards the flock center

\section{III.A. Mate Selection Algorithm}

The mate selection algorithm is used to achieve objective 1 and is given in Algorithm 1. The algorithm first computes a matrix, $d$, where the $i^{t h}$ row is a vector of distances from agent $i$ to each other agent. Mates are assigned based on the minimum value in each row. To avoid any agents choosing themselves as mates (since the distance from an agent to itself is zero), the diagonal elements of $d$ are set to $\infty$.

Next, a vector, $m$, of length $n$ is initialized with all zeros. Beginning with agent 1 , mates are selected by finding the column of $d$ that corresponds to the minimum element in the row of the current agent (agent $i$ ). The $i^{t h}$ element of $m$ is assigned this index. $m$ keeps track of which agents are mated, i.e. agent $i$ is mated to agent $m_{i}$. The $m_{i}^{t h}$ element of $m$ is then assigned to $i$. This process only takes place if $m_{i}=0$ so that each agent is assigned a mate only once.

Next, the $i^{\text {th }}$ and $m_{i}^{t h}$ columns of $d$ are assigned values of $\infty$. This ensures that no other agents will select agents $i$ or $m_{i}$ as mates. Thus, Algorithm 1 ensures that $n / 2$ unique pairs of agents are selected, satisfying objective 1.

\section{III.B. Potential Field Definition}

We will design a potential field, $V\left(r_{i}\right)$, associated with agent $i$ to achieve objective 2 . The potential field will have three components - one to attract the agent towards its mate, one to repel the agent away from non-mate agents, and one to drive the agent towards the flock center. Let the attraction component be

$$
V_{1}\left(r_{i}\right)=\frac{1}{2} W_{a}\left\|r_{i}-r_{j}\right\|^{2}
$$

where $W_{a}$ is the weight given to the attraction objective. Let the repelling component be

$$
V_{2}\left(r_{i}\right)=\frac{1}{2} W_{r} \sum_{k \neq i, k \neq j}^{n}\left\|r_{i}-r_{j}\right\|^{-2}
$$




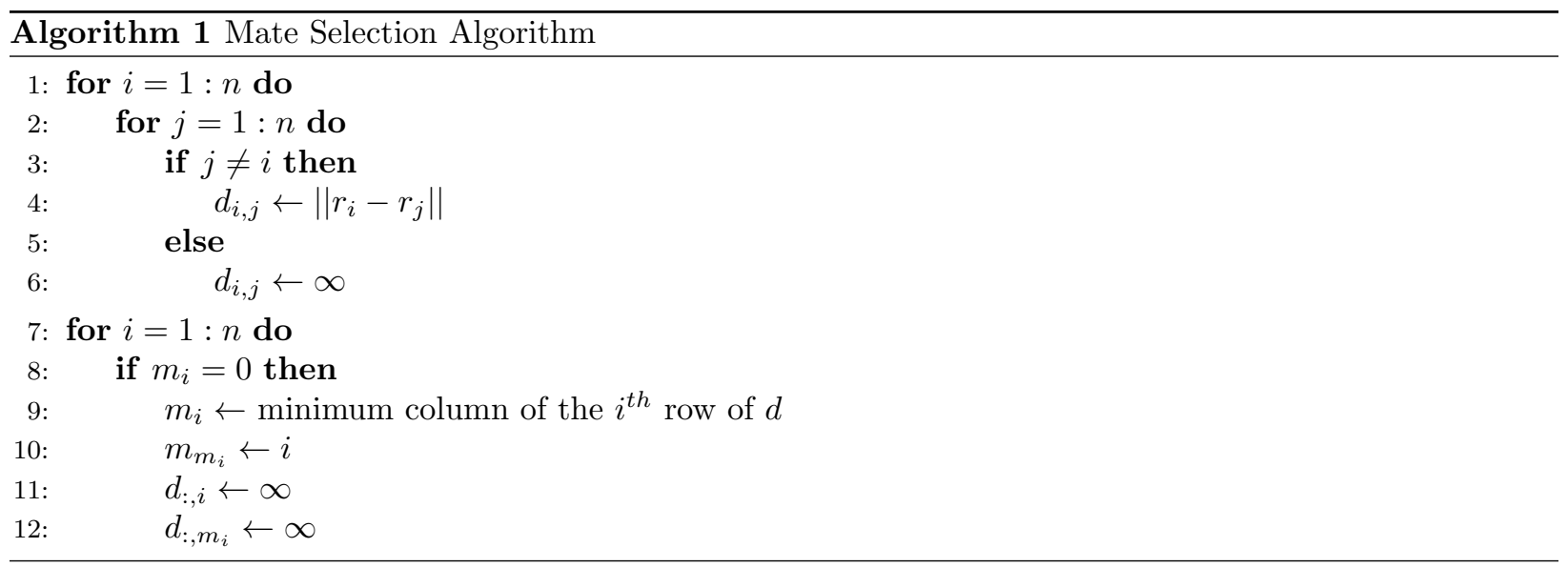

where $W_{r}$ is the weight given to the repelling component. Finally, the flock-centering component is

$$
V_{3}\left(r_{i}\right)=\frac{1}{2} W_{f}\left\|r_{i}-c\right\|^{2}
$$

where $W_{f}$ is the weight given to the flocking component and where $c=\left[c_{x} c_{y}\right]^{\top}=\frac{\sum_{i}^{n} r_{i}}{n}$ is the center of the flock. The total potential is $V=V_{1}+V_{2}+V_{3}$. All three components of the potential are positive semidefinite and differentiable. This allows us to use the potential field gradient in the control law to minimize the potential.

An example contour map of this potential field is shown in Figure 1. The red diamond and blue discs on the plot represent the agents in the system. The potential field shown is from the perspective of the agent plotted as a red diamond. On this contour plot, blue represents low potential and yellow represents high potential. Note that the potential tends toward infinity as position moves towards an obstacle agent. The potential in the vicinity of obstacles has been saturated in this plot. Otherwise, we would see contours placed close together around the obstacle agents.

In this example, the red diamond agent has selected the agent at $(1.8,3.2)$ (the disc closest to itself) as its mate. The minimum potential lies somewhere between the red agent and its mate due to the attractive and flocking components. The minimum is not found exactly at the location of the mate because the flocking component influences the red agent toward the centroid of the flock. We can see that the potential gradually increases as the red agent moves away from the flock, and sharply increases as it moves towards obstacle agents.

\section{III.C. Outer-Loop Control Law}

The vehicle will have achieved all of its goals when its potential, $V$, is minimized. Therefore, we design a velocity command, $v_{i_{c m d}}$ to be in the direction of steepest descent in the potential field,

$$
v_{i_{c m d}}=-K \nabla V\left(r_{i}\right)
$$

where $K$ is a control gain. This velocity will always be in direction of decreasing potential. By differentiating (11), (12), and (13), we obtain

$$
\nabla V\left(r_{i}\right)=W_{a}\left(r_{i}-r_{j}\right)-W_{r} \sum_{k \neq i, k \neq j}^{n}\left[\left(r_{i}-r_{k}\right)^{\top}\left(r_{i}-r_{k}\right)\right]^{-2}\left(r_{i}-r_{k}\right)+W_{f}\left(r_{i}-c\right)
$$

Next, we must specify $u_{i}$ so that agent $i$ 's velocity tracks $v_{i_{c m d}}$. This will be done via a saturated 


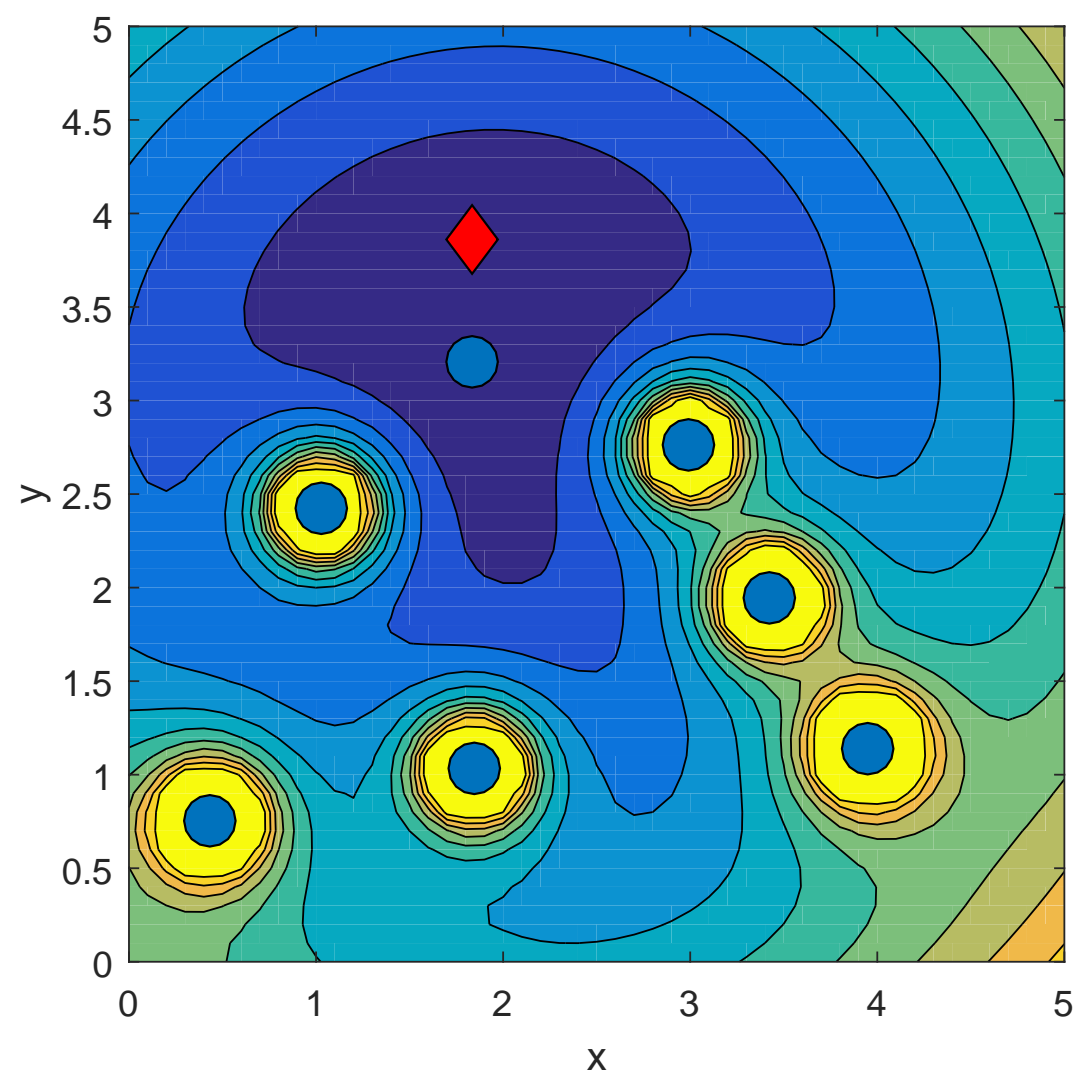

Figure 1: Example potential field experienced by the red diamond agent

proportional feedback controller

$$
u_{i}= \begin{cases}u_{\max } \frac{v_{i_{c m d}}-\dot{r}_{i}}{\left\|v_{i_{c m d}}-\dot{r}_{i}\right\|} & \text { if }\left\|K_{V}\left(v_{i_{c m d}}-\dot{r}_{i}\right)\right\|>u_{\max } \\ K_{v}\left(v_{i_{c m d}}-\dot{r}_{i}\right) & \text { otherwise }\end{cases}
$$

where $u_{\max }$ is the maximum allowable acceleration and $K_{v}$ is a control gain. The saturation is required because the vehicle's motors have a maximum thrust output. $u_{\max }$ should be chosen conservatively so that large attitude commands are not generated.

In the following, we will analyze the stability of the linear part of the velocity controller in (16). Let $v_{i}=\dot{r}_{i}$. We can find the transfer function from $v_{i_{c m d}}$ to $v$ by using (16) to rewrite (1) in the Laplace-domain as

$$
s v_{i}=K_{v}\left(v_{i_{c m d}}-v_{i}\right)
$$

where $s$ is the Laplace-domain variable. Rewriting (17) in transfer function form yields

$$
\frac{v_{i}}{v_{i_{c m d}}}=\frac{K_{v}}{s+K_{v}}
$$

(18) is a first order low-pass filter with bandwidth of $K_{v}$. This means that $v_{i}$ will always converge to $v_{i_{c m d}}$ with a time-constant of $1 / K_{v}$. $K_{v}$ should be chosen to be large enough that there is significant time-scale separation between the rate of change of $v_{i_{c m d}}$ and the time-constant of (18). In other words, the velocity-tracking controller should be fast enough so that the commanded velocity value is reached before it changes significantly. In practice, the magnitude of $K_{v}$ is limited by effects of amplifying sensor noise and uncertainties. If this criterion causes the response time of velocity controller to be similar to the 
rate of change of the velocity command, then a more sophisticated velocity controller is necessary, such as proportional-integral-derivative (PID) or adaptive control.

\section{III.D. Inner-Loop Control Law}

The commanded Euler angles generated by $(7,8)$ are controlled by an inner-loop controller, which can take many forms, such as the attitude controller given in. ${ }^{22}$ Most often, a set of simple PID controllers is sufficient for inner-loop control of a multi-rotor aircraft. We will implement PID attitude control as follows

$$
\begin{aligned}
\tau_{1} & =K_{p_{\phi}}\left(\phi_{c m d}-\phi\right)+K_{i_{\phi}} \int_{0}^{t}\left(\phi_{c m d}-\phi\right) d \bar{t}+K_{d_{\phi}} \frac{d}{d t}\left(\phi_{c m d}-\phi\right) \\
\tau_{2} & =K_{p_{\theta}}\left(\theta_{c m d}-\theta\right)+K_{i_{\theta}} \int_{0}^{t}\left(\theta_{c m d}-\theta\right) d \bar{t}+K_{d_{\theta}} \frac{d}{d t}\left(\theta_{c m d}-\theta\right)
\end{aligned}
$$

where $K_{p_{\phi}}, K_{i_{\phi}}$, and $K_{d_{\phi}}$ are the proportional, integral, and derivative gains respectively for roll, $K_{p_{\theta}}, K_{i_{\theta}}$, and $K_{d_{\theta}}$ are the proportional, integral, and derivative gains respectively for pitch, and $\bar{t}$ is a dummy variable.

Note that here we are ignoring the dynamics of the motors of the aircraft. This means we assume we can always generate the desired thrust and torques at the instant they are commanded. The motors require their own controllers to ensure that they are providing the commanded thrust and torques, but for the purposes of this paper, we will ignore this aspect of the vehicle design. This is a reasonable assumption because motor dynamics and vehicle dynamics operate on much different time-scales, i.e., the motor response should always be much faster than the angular and translational responses of the vehicle.

Another aspect of the design that is ignored is yaw control. In practice, the mating vehicles must yaw such that their linking interfaces face one another. This is a trivial problem that can be solved by applying a gain to the yaw angle error between the two linking interfaces to generate $\tau_{3}$. For a more concise presentation, we will assume that the vehicles are capable of linking to each other from any direction, and thus $\tau_{3} \triangleq 0$.

\section{Simulation}

A simulation was performed for 16 vehicles. This results in 4 linking phases. Each linking phase was considered complete when the distance between mated pairs became less than or equal to a parameter, $\delta$. The 16 agents were initialized at the same altitude with randomized $(x, y)$ positions in a $100 \mathrm{~m} \times 100 \mathrm{~m}$ grid and with 0 velocities. The moment of inertia matrix for each vehicle was a diagonal matrix,

$$
I=\left[\begin{array}{ccc}
I_{x} & 0 & 0 \\
0 & I_{y} & 0 \\
0 & 0 & I_{z}
\end{array}\right]
$$

where $I_{x}, I_{y}$, and $I_{z}$ are the moments of inertia about the $x, y$ and $z$-axes respectively. The simulation parameters are shown in Table 1.

Table 1: Simulation parameters

\begin{tabular}{|r|l|r|l|r|l|}
\hline Parameter & Value & Parameter & Value & Parameter & Value \\
\hline$W_{a}$ & 1 & $m$ & $1.3 \mathrm{~kg}$ & $K_{p_{\phi}}$ & 0.1 \\
\hline$W_{r}$ & 500 & $g$ & $9.81 \mathrm{~m} / \mathrm{s}^{2}$ & $K_{i_{\phi}}$ & 0 \\
\hline$W_{f}$ & 0.5 & $I_{x}$ & $8.1 \times 10^{-} 3 \mathrm{kgm}^{2}$ & $K_{d_{\phi}}$ & 0.1 \\
\hline$K$ & 0.1 & $I_{y}$ & $8.1 \times 10^{-} 3 \mathrm{kgm}^{2}$ & $K_{p_{\theta}}$ & 0.1 \\
\hline$K_{v}$ & 10 & $I_{z}$ & $14.2 \times 10^{-} 3 \mathrm{kgm}^{2}$ & $K_{i_{\theta}}$ & 0 \\
\hline$u_{\max }$ & $1.7 \mathrm{~m} / \mathrm{s}^{2}$ & $\delta$ & $0.05 \mathrm{~m}$ & $K_{d_{\theta}}$ & 0.1 \\
\hline
\end{tabular}


Figure 2 shows the positions and velocity commands of each agent at the start of each phase. Figure $2 \mathrm{a}$ shows the first phase, Figure $2 \mathrm{~b}$ shows the second phase, Figure $2 \mathrm{c}$ shows the third phase, and Figure $2 \mathrm{~d}$ shows the fourth phase. Each blue marker on the plots represents an agent, and the red arrows are the velocity command vectors of the agents they are attached to. We can see that for most agents, the largest components of their velocities point towards their mate. In Figure 2a, some agents located close to the edge of the group have velocity commands with larger components pointing toward the center of the flock because they are further away from that center than the rest of the agents.

Figure 3 shows the potential field values for each agent during the simulation. Figures 3a, 3b, 3c, and 3d show the first, second, third, and fourth linking phases, respectively. We can see that the potential of every agent mostly decreases over time and converges before the start of the next phase. Potential can increase when the point of minimum potential is moving faster than the agent. This can occur during collision avoidance maneuvers. From Figure 3b, we can see that such an event took place with Agents 6 (light blue) and 8 (dark blue) during the second linking phase because their potentials began increasing after 36 seconds.

Eventually, the potential converges to 0 at the end of the final linking phase. There are discrete jumps in potential when the current phase completes and the next one start because the mate selection process takes place between phases. This causes the potential field to change completely. Furthermore, note that once two agents link, they are considered to be a single agent in the subsequent phase.

Figure 4 shows the distances between the mating pairs for each phase of the linking. Figures $4 \mathrm{a}, 4 \mathrm{~b}, 4 \mathrm{c}$, and $4 \mathrm{~d}$ show these values for the first, second, third, and fourth phases respectively. The legends in Figure 4 indicate which agents are paired. We can see that the distances between all pairs converge to less than $\delta$ before each subsequent phase is begun. Note that no mating errors increased throughout the simulation, although such an event is possible due to the collision avoidance term of the potential field.

\section{Conclusions and Future Work}

This paper has presented a potential field guidance algorithm for in-flight linking of multi-rotor aircraft. This investigation was performed as a part of NASA's Link! project. Through analysis and simulation, the guidance algorithm presented here has been identified as plausible for a multi-agent system of multi-rotor vehicles. The vehicles in a simulation with randomized initial conditions were all able to link together by finding the minimum potential.

A requirement of the potential field guidance algorithm is that every agent has access to a position measurement of itself and every other agent in the system. The sensors and communication abilities on board each vehicle will need to ensure that this is possible. This is a potential drawback to the presented approach since communication bandwidth will be limited, and the required data transfer increases with the square of the number of vehicles. This limitation may be circumvented by using on-board sensors to estimate the positions of the other vehicles. However, such sensors will limit the range of group.

In future work, the guidance algorithm presented here will be implemented on a system of multirotor vehicles, and flight experiments will be performed. To meet this goal, the necessary sensors, communication, and state estimation schemes must be investigated. Some examples of sensors that will be investigated are GPS, computer vision, ${ }^{23,24}$ LIDAR, ${ }^{25}$ and ultra-wideband radios for close-range localization of member vehicles. ${ }^{26}$ Another aspect of implementation that must be addressed is the design of a linking mechanism that can be outfitted onboard the vehicles and the switching of control allocation amongst the various motors once 2 single agents are linked to form a new larger agent with twice as many engines.

\section{References}

\footnotetext{
${ }^{1}$ Noll, T. E., Brown, J. M., Perez-Davis, M. E., Ishmael, S. D., Tiffany, G. C., and Gaier, M., "Investigation of the Helios Prototype Aircraft Mishap," Mishap report, NASA, 2004.

${ }^{2}$ Anderson, C., "Dangerous Experiments: Wingtip Coupling at 15,000 Feet," Flight Journal, Vol. 5, No. 6, 2000 , pp. 64.

${ }^{3}$ Miller, J., "Project Tom-Tom," Aerophile, Vol. 1, 1977, pp. 161-164.

${ }^{4}$ Moore, J. and Maddalon, D., "Multibody Transport Concept," 2nd International Very Large Vehicles Conference, 1982, p. 810 .
} 


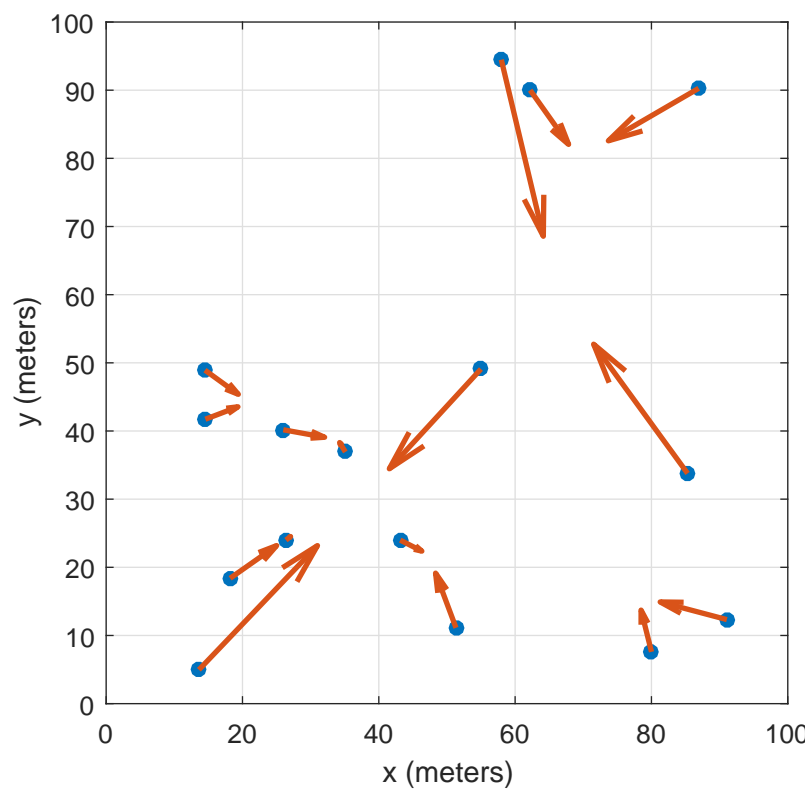

(a) Agent positions and velocity commands at the start of phase 1

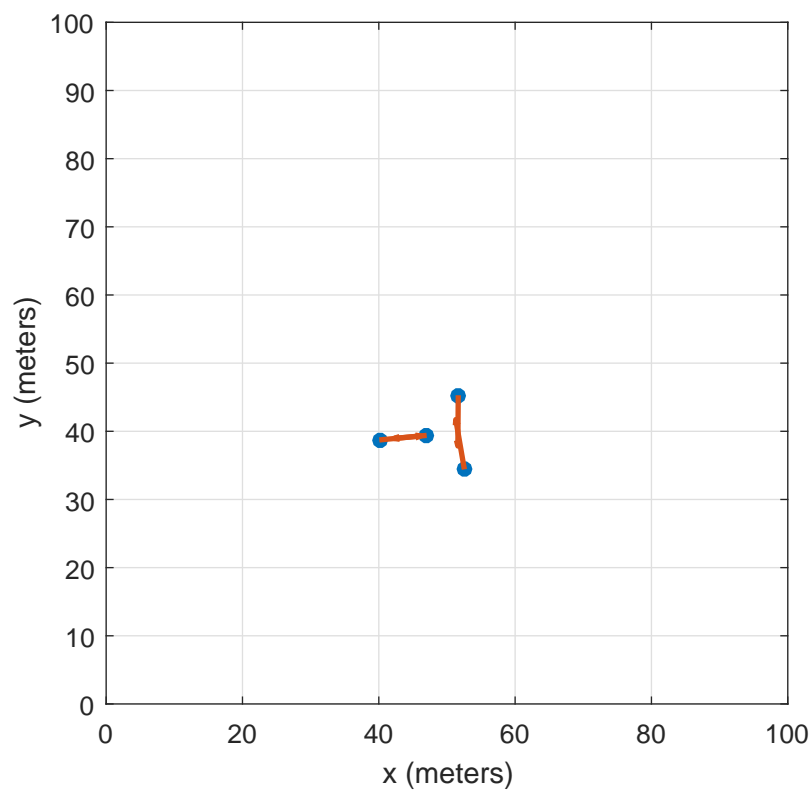

(c) Agent positions and velocity commands at the start of phase 3

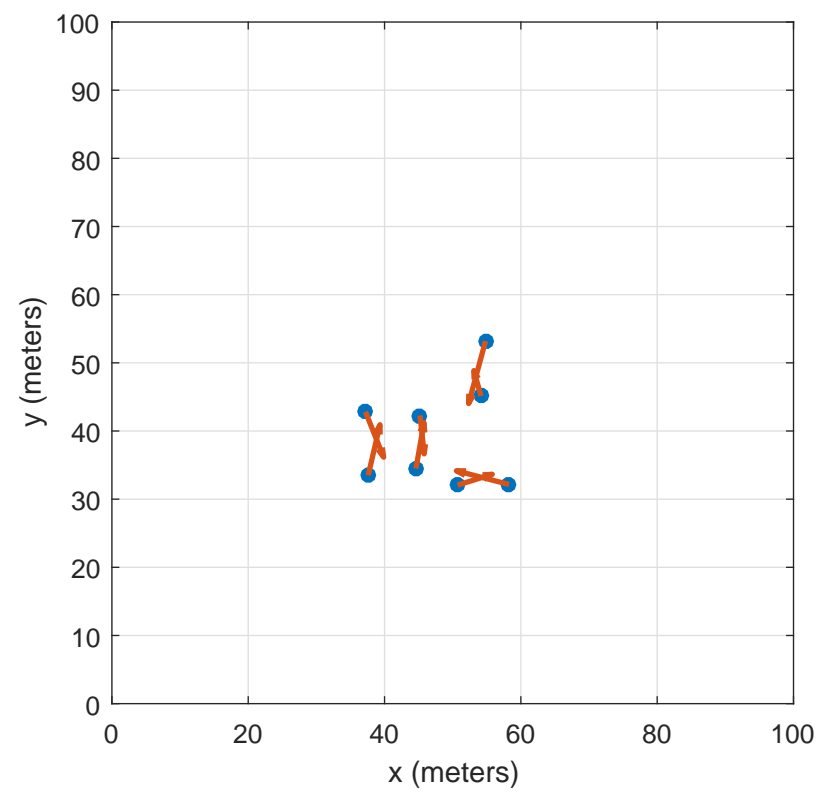

(b) Agent positions and velocity commands at the start of phase 2

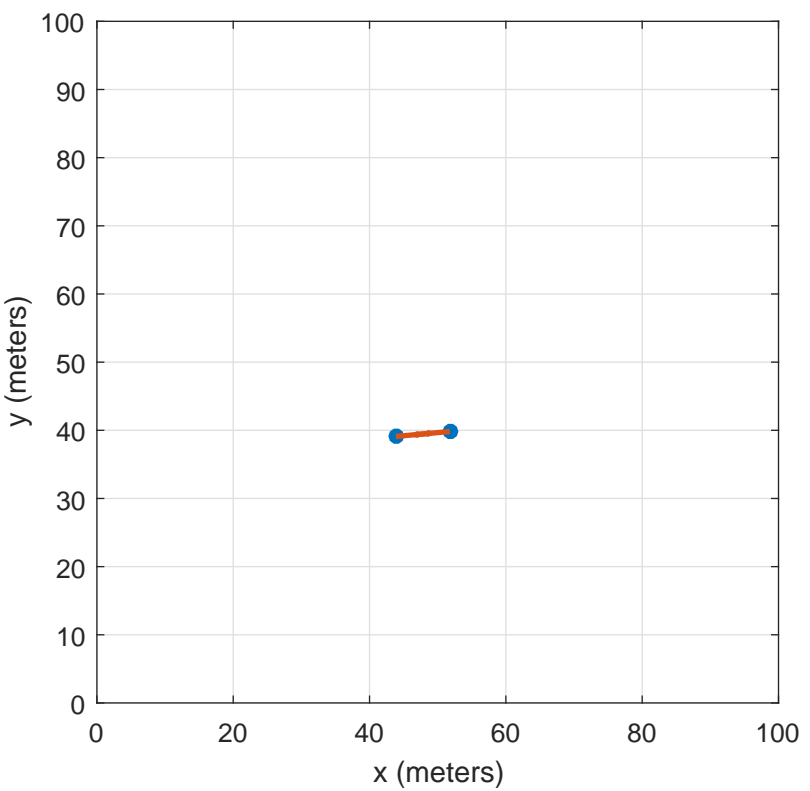

(d) Agent positions and velocity commands at the start of phase 4

Figure 2: Agent positions and velocity commands at the start of each linking phase

${ }^{5}$ Magill, S. A. and Durham, W. C., "Modelling and Simulation of Wingtip-Docked Flight," moment, Vol. 1000, 2002, pp. 2 .

${ }^{6}$ Magill, S. A., Schetz, J. A., and Mason, W. H., "Compound Aircraft Transport: A Comparison of Wing Tip-Docked and Close-Formation Flight," 41st Aerospace Sciences Meeting and Exhibit, 2003, pp. 2003-0607.

${ }^{7}$ Casal, A. and Yim, M. H., "Self-Reconfiguration Planning for a Class of Modular Robots," Photonics East'99, International Society for Optics and Photonics, 1999, pp. 246-257. 


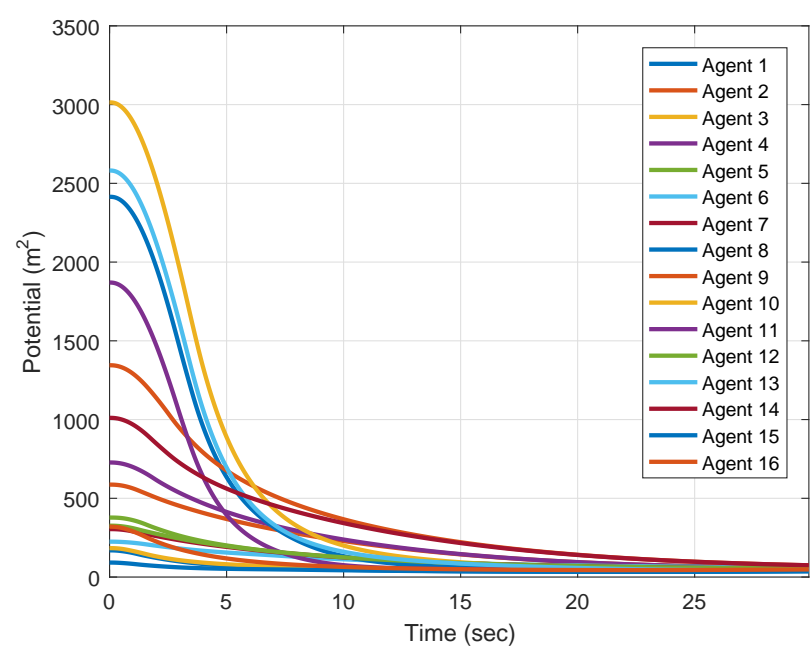

(a) Potential values over time for each agent during the first linking phase

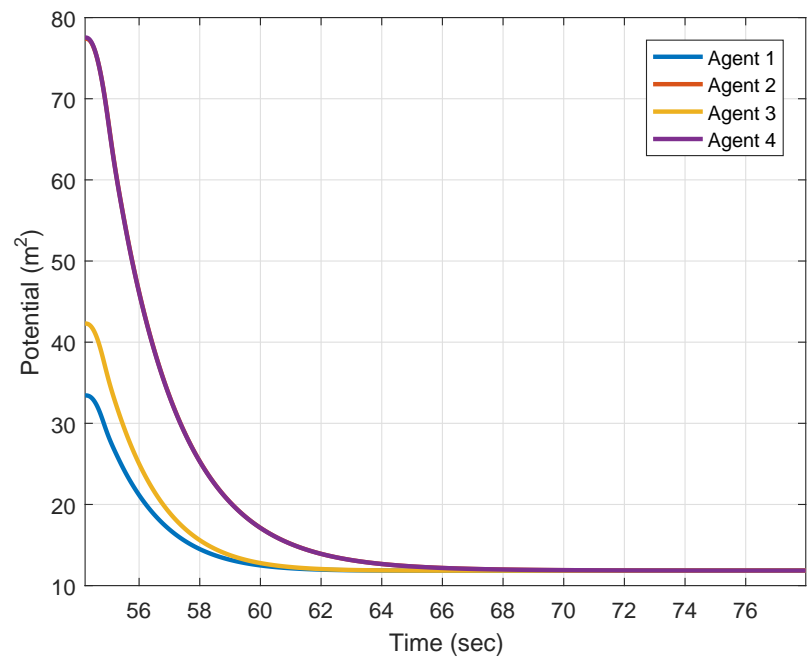

(c) Potential values over time the four agents present during the third linking phase

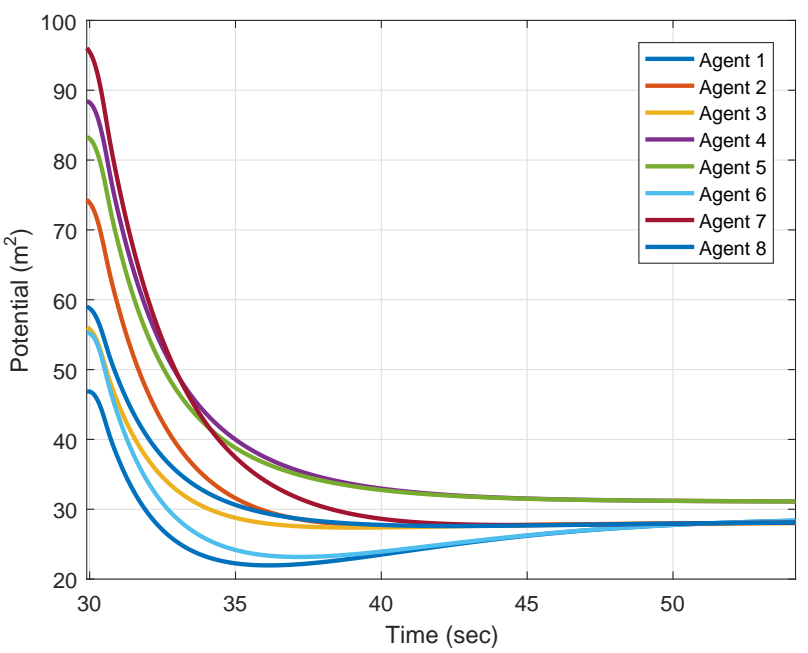

(b) Potential values over time for each agent during the second linking phase

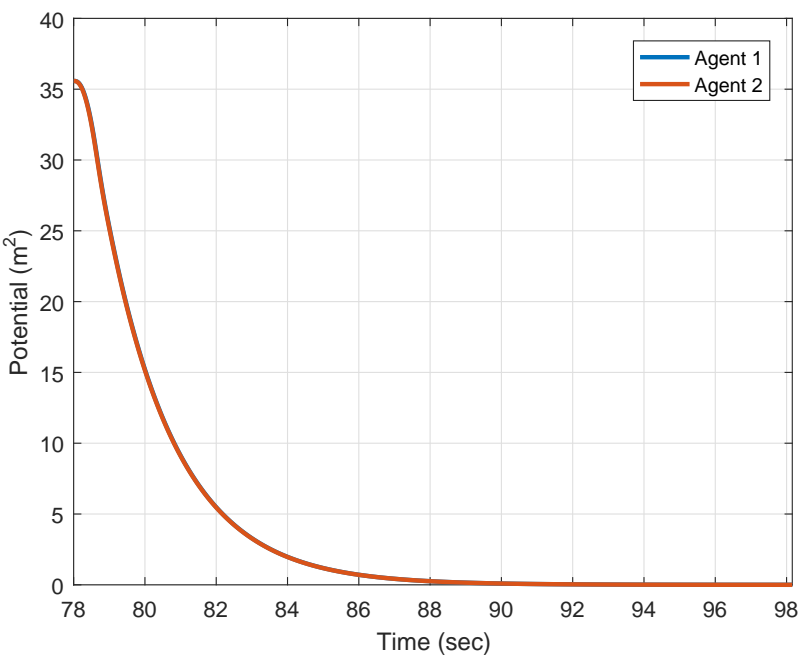

(d) Potential values over time the two remaining agents during the final linking phase

Figure 3: Potential values for each agent throughout the simulation

${ }^{8}$ Yim, M., Zhang, Y., and Duff, D., "Modular Robots," Spectrum, IEEE, Vol. 39, No. 2, 2002, pp. 30-34.

${ }^{9}$ Vassilvitskii, S., Yim, M., and Suh, J., "A Complete, Local and Parallel Reconfiguration Algorithm for Cube Style Modular Robots," Proceedings of IEEE International Conference on Robotics and Automation, Vol. 1, IEEE, 2002, pp. 117122 .

${ }^{10}$ White, P., Zykov, V., Bongard, J. C., and Lipson, H., "Three Dimensional Stochastic Reconfiguration of Modular Robots." Robotics: Science and Systems, Cambridge, 2005, pp. 161-168.

${ }^{11}$ Oung, R. and DAndrea, R., "The Distributed Flight Array," Mechatronics, Vol. 21, No. 6, 2011, pp. $908-917$.

${ }^{12}$ Montalvo, C. and Costello, M., "Meta Aircraft Flight Dynamics," Journal of Aircraft, Vol. 52, No. 1, 2014, pp. 107-115.

${ }^{13}$ Troub, B. and Montalvo, C., "Meta Aircraft Controllability," AIAA Atmospheric Flight Mechanics Conference, 2016, p. 3395.

${ }^{14}$ Montalvo, C., Meta Aircraft Flight Dynamics and Controls, Ph.D. thesis, Georgia Institute of Technology, 2014.

${ }^{15}$ Reynolds, C. W., "Flocks, Herds and Schools: A Distributed Behavioral Model," ACM SIGGRAPH Computer Graphics, Vol. 21, No. 4, 1987, pp. 25-34. 


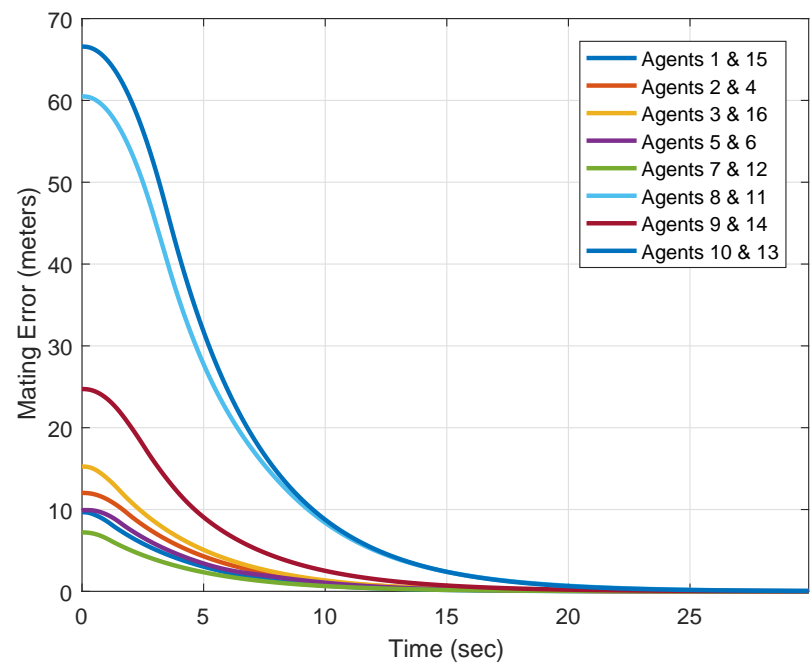

(a) Distances between the 8 pairs during the first linking phase

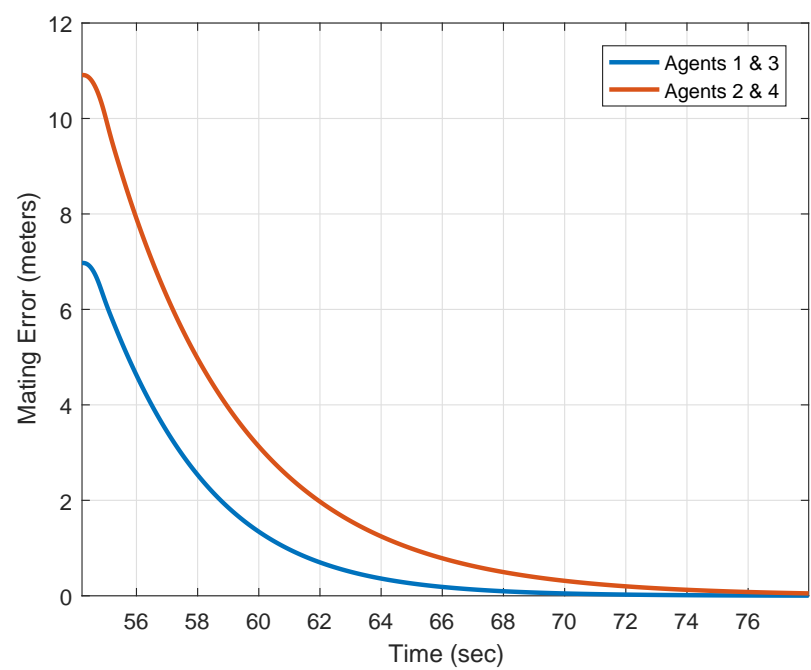

(c) Distance between the 2 pairs during the third linking phase

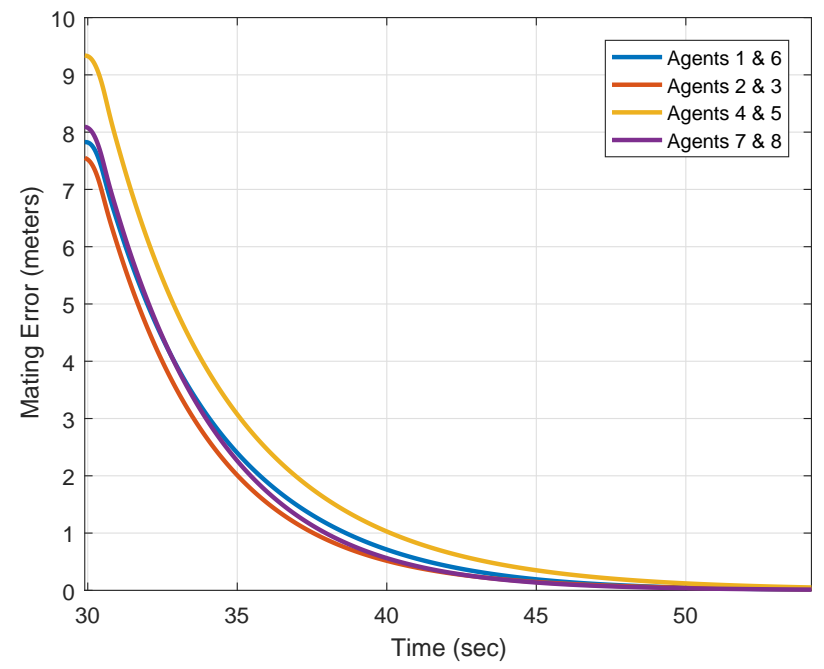

(b) Distances between the 4 pairs during the second linking phase

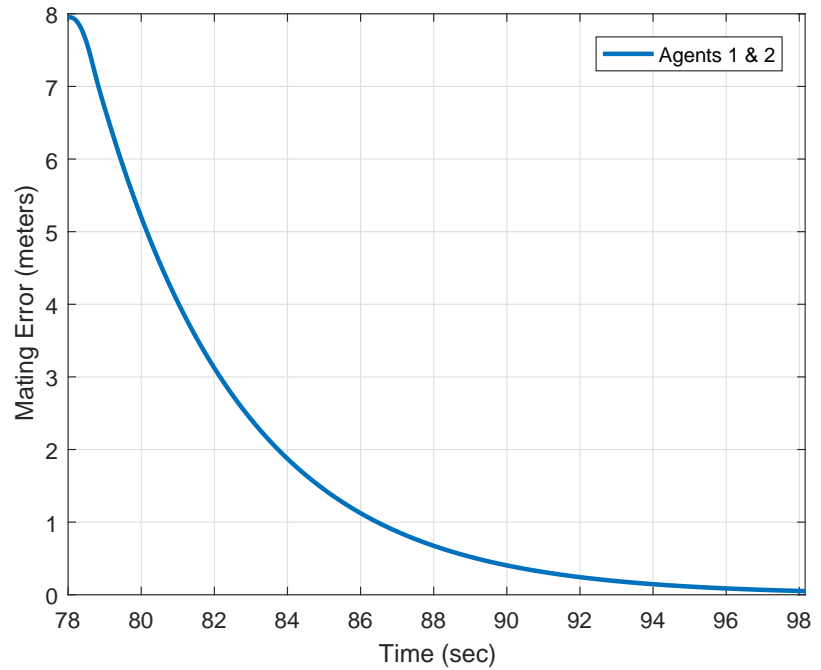

(d) Distance between the remaining pair during the final linking phase

Figure 4: Distance between mates for each linking phase

\footnotetext{
${ }^{16}$ Olfati-Saber, R., "Flocking for Multi-Agent Dynamic Systems: Algorithms and Theory," IEEE Transactions on Automatic Control, Vol. 51, No. 3, 2006, pp. 401-420.

${ }^{17}$ Tanner, H. G., Jadbabaie, A., and Pappas, G. J., "Stable Flocking of Mobile Agents, Part I: Fixed Topology," Proceedings of the 42nd IEEE Conference on Decision and Control, Vol. 2, IEEE, 2003, pp. 2010-2015.

${ }^{18}$ Tanner, H. G., Jadbabaie, A., and Pappas, G. J., "Stable Flocking of Mobile Agents Part II: Dynamic Topology," Proceedings of the 42nd IEEE Conference on Decision and Control, Vol. 2, IEEE, 2003, pp. 2016-2021.

${ }^{19}$ Tanner, H. G., Jadbabaie, A., and Pappas, G. J., "Flocking in Fixed and Switching Networks," IEEE Transactions on Automatic Control, Vol. 52, No. 5, 2007, pp. 863-868.

${ }^{20} \mathrm{Su}, \mathrm{H}$., Wang, X., and Lin, Z., "Flocking of Multi-Agents with a Virtual Leader," IEEE Transactions on Automatic Control, Vol. 54, No. 2, 2009, pp. 293-307.

${ }^{21}$ Stevens, B. L., Lewis, F. L., and Johnson, E. N., Aircraft Control and Simulation: Dynamics, Controls Design, and Autonomous Systems, John Wiley \& Sons, 2015.
} 
${ }^{22}$ Mahony, R., Kumar, V., and Corke, P., "Multirotor Aerial Vehicles: Modeling, Estimation, and Control of Quadrotor," IEEE Robotics \&S Automation Magazine, , No. 19, 2012, pp. 20-32.

${ }^{23}$ Forster, C., Pizzoli, M., and Scaramuzza, D., "SVO: Fast Semi-Direct Monocular Visual Odometry," Proceedings of the IEEE International Conference on Robotics and Automation, IEEE, 2014, pp. 15-22.

${ }^{24}$ Rodríguez-Canosa, G. R., Thomas, S., del Cerro, J., Barrientos, A., and MacDonald, B., "A Real-Time Method to Detect and Track Moving Objects (DATMO) From Unmanned Aerial Vehicles (UAVs) Using a Single Camera," Remote Sensing, Vol. 4, No. 4, 2012, pp. 1090-1111.

${ }^{25}$ Russ, M., Vohla, M., Stütz, P., and OYoung, S., "LIDAR-Based Object Detection on Small UAV: Integration, Experimentation and Results," AIAA Infotech Conference, 2012.

${ }^{26}$ Gezici, S., Tian, Z., Giannakis, G. B., Kobayashi, H., Molisch, A. F., Poor, H. V., and Sahinoglu, Z., "Localization via Ultra-Wideband Radios: A Look at Positioning Aspects for Future Sensor Networks," Signal Processing Magazine, IEEE, Vol. 22, No. 4, 2005, pp. 70-84. 